\title{
CONTENIDO EN GRASA INTRAMUSCULAR DEL LOMO Y LA PALETA DE CERDOS IBÉRICOS SEGÚN SU SISTEMA DE ALIMENTACIÓN Y MANEJO
}

\section{INTRAMUSCULAR FAT CONTENT IN LOIN AND SHOULDER OF IBERIAN PIGS ACCORDING TO THE DIET AND REARING SYSTEM}

\author{
Guadalupe Quintanilla Caballo y Beatriz Isabel Redondo
}

Dpto. Producción Animal. Facultad de Veterinaria UCM. Madrid, España.

\section{RESUMEN}

La calidad de los productos curados obtenidos del cerdo ibérico, está directamente relacionada con la raza, edad y sistema de producción de los animales. El presente trabajo estudia el contenido en grasa intramuscular y la composición en ácidos grasos de paletas y lomos curados de cerdo ibérico según el sistema de alimentación utilizado. Para la consecución de nuestro objetivo se utilizaron 24 hembras ibéricas castradas (ovariectomizadas) de 10 meses de edad divididas en dos grupos experimentales durante la fase final de engorde. El primer grupo (12 cerdas) fue alimentado en un sistema "ad libitum" en intensivo con pienso concentrado rico en ácido oleico; mientras que el segundo grupo fue alimentado con hierba y bellotas en el sistema tradicional denominado "montanera". El porcentaje de ácido palmítico fue superior en paletas y lomos curados para el grupo alimentado con pienso; mientras que el ácido oleico en lomos y el ácido linoleico en paletas fueron mayores en los animales de montanera. El porcentaje de grasa intramuscular fue superior en el grupo de montanera. El presente estudio confirma la gran influencia que tiene la alimentación del animal en la composición de los productos curados obtenidos del cerdo ibérico.

Palabras clave: ácidos grasos, cerdo ibérico, grasa intramuscular, montanera. 


\begin{abstract}
The quality of cured products obtained from the Iberian pigs is directly related with the breed, age and type of production system of the animals. The aim of the present work was to study the differences of fatty acids composition and intramuscular fat content in dry-cured shoulders and loins of Iberian pigs depends on diet and rearing system. For that purpose, 24 Iberian castrated (ovariectomized) females of 10 months age were divided into two experimental groups during the finishing period. The first group (12 pigs) was raised in an intensive system "ad libitum" with concentrate feeds rich in oleic acid; while the second group was raised in an extensive traditional system called "montanera" fed with acorns and grass. The palmitic acid was higher in those dry-cured shoulders and loins from pigs reared with concentrate feeds; while the oleic acid in loins and linoleic acid in shoulders were higher in those pigs reared in montanera. Intramuscular fat percentage was higher for pigs raised in montanera, feeding by acorns and grass, than in pigs fed by concentrated diets. This study demonstrated the high influence of animal feeding in the final composition of the dry-cured products obtained from the Iberian pig.
\end{abstract}

Key words: fatty acids, intramuscular fat, Iberian pig, montanera.

\title{
INTRODUCCIÓN
}

El cerdo ibérico es una raza rústica criada tradicionalmente en el sur-oeste de la Península Ibérica. El ecosistema de la dehesa constituye su hábitat natural, se trata de una producción en extensivo que ha perdurado durante siglos. Aunque, a día de hoy, se siguen manteniendo los sistemas tradicionales extensivos para su explotación, también proliferan los sistemas intensivos y semiextensivos.

En la actualidad, la explotación extensiva del cerdo ibérico se desarrolla en la dehesa, donde la producción de bellotas depende de numerosos factores como el clima, la especie o el manejo, y varía entre 3500 y $12000 \mathrm{~kg}$ bellota/ha al año (Vázquez et al., 2000). Tradicionalmente, los sistemas de producción de porcino en la dehesa constan de varias fases: cría, recría y engorde en cebo o montanera, que constituyen el ciclo completo del porcino ibérico, siendo la fase de cebo en montanera la que caracteriza el proceso productivo extensivo (Aparicio, 1987). La montanera transcurre durante los meses de otoño-invierno, coincidiendo con la época de producción de bellota en las dehesas, por lo que el sistema de 
producción está íntimamente ligado al aprovechamiento de los recursos naturales por parte del cerdo ibérico.

El jamón, seguido de la paleta y el lomo de cerdo ibérico, son los productos más apreciados por los consumidores. Existen distintas clasificaciones comerciales para estos productos curados en función del sistema de cría empleado, lo que determina diferentes precios para su comercialización. Las denominaciones comerciales de los productos del cerdo ibérico según su raza, manejo y alimentación están reguladas bajo el artículo 3 del RD 1469/2007.

Estudios anteriores han demostrado que el cerdo ibérico alimentado en montanera ingiere grandes cantidades de ácidos grasos monoinsaturados (AGM), triglicéridos y fracciones de fosfolípidos procedentes de las bellotas (Rey et al., 1997), teniendo así un menor contenido en ácidos grasos poliinsaturados (AGP) que los alimentados con dietas comerciales (Cava et al., 1997). Así mismo, la alimentación a base de pastos y bellotas produce un incremento en la concentración de antioxidantes naturales (tocoferoles) consiguiendo una mayor estabilidad oxidativa de la carne (Rey et al., 1997).

La jugosidad de la carne y de los productos curados está determinada por dos factores, el contenido en agua y la grasa intramuscular, por lo que aquellos productos curados que tengan una humedad baja, la jugosidad será principalmente afectada por el contenido en grasa intramuscular (López-Bote, 2000). El contenido y composición de la grasa intramuscular en el cerdo determina en una amplia proporción la calidad sensorial de la carne, proporcionando sapidez, aroma, sabor y jugosidad (López-Bote, 2000).

Otro aspecto muy estudiado en los últimos años es la composición en ácidos grasos del tejido adiposo subcutáneo, debido a su repercusión en la calidad de los productos del cerdo ibérico y su utilización como criterio objetivo para la clasificación y pago de las canales, hasta la publicación de la norma de calidad del ibérico (RD 1469/2007). La utilización de la determinación de los ácidos grasos en el tejido subcutáneo dio lugar a la aparición de piensos compuestos que trataban de imitar la composición en ácidos grasos de la bellota y que incorporaban grandes cantidades de grasa en su formulación, si bien la composición de la bellota depende de la climatología, del arbolado específico de cada dehesa y sus cuidados (Isabel y González, 2008) por lo que las características de los productos derivados de cerdos ibéricos criados en montanera son difíciles, por no decir, casi imposibles de imitar en su totalidad. 
El objetivo de nuestro trabajo fue determinar el contenido en grasa intramuscular y la composición de ácidos grasos en paletas y lomos de cerdos ibéricos, comparando cerdos que han sido engordados a base de bellota y otros recursos naturales que proporciona la dehesa (cerdos en montanera), con otros cerdos ibéricos alimentados con un pienso rico en ácido oleico en condiciones intensivas.

\section{MATERIAL Y MÉTODOS}

Para nuestro estudio se utilizaron 24 hembras ibéricas castradas que se dividieron en dos grupos experimentales de 12 animales cada uno, montanera y cebo. Estos animales provenían de una explotación situada en Higuera de Vargas (Badajoz), con 10 meses de edad y una media de $81,8 \mathrm{~kg}$ de peso vivo. Las cerdas fueron alimentadas de dos formas, por un lado, las cerdas de cebo fueron alimentadas ad libitum con un pienso comercial (rico en ácido oleico, con $14 \%$ de PB, 7,4\% GB, grasa bruta y $3250 \mathrm{kcal}$ de EM) para la fase de engorde, mientras que las cerdas en sistema de montanera fueron alimentadas con hierba y bellota siguiendo el sistema tradicional. La composición tanto del pienso como de la bellota y de la hierba, con los que fueron alimentados los animales experimentales, se puede observar en la Tabla 1.

Los cerdos fueron sacrificados tras la etapa de engorde (4 meses) con una media de $162,9 \mathrm{~kg}$ de peso vivo en el matadero el Mayorazgo S.L. (Jabugo- Huelva). Tras el sacrificio, se obtuvieron los lomos y las paletas de los mismos y fueron sometidos a un proceso de curación tradicional. Para ello, los lomos fueron sazonados frotándolos con una mezcla de sal, nitritos, aceite de oliva y especias como pimentón (Capsicumannuum, L.), orégano (Origanumvulgare L.) y ajo (Altiumsativum, L.). Se mantuvieron a $4^{\circ} \mathrm{C}$ durante 4 días para facilitar la penetración de la mezcla. Después, los lomos se introdujeron en tripas de colágeno y se mantuvieron a $4^{\circ} \mathrm{C}$ con una humedad relativa del $80 \%$ durante 30 días. Por último, se aumentó la temperatura a $12-16^{\circ} \mathrm{C}$ y se disminuyó la humedad relativa a $70 \%$ durante 3 meses. Para la producción de paletas curadas, éstas se sometieron a un proceso de salado y después se mantuvieron con temperaturas entre 0 y $3^{\circ} \mathrm{C}$ y $80-90 \%$ de humedad relativa durante 6 meses. Después, las paletas se almacenaron a $10-25^{\circ} \mathrm{C}$ y $60-80 \%$ de humedad relativa durante 12 meses. Este proceso se extendió hasta un total de 18 meses. Las muestras se recogieron al final del periodo de curación y se conservaron envasadas al vacío y a $-20^{\circ} \mathrm{C}$ hasta la determinación de los diferentes compuestos. 
Tabla 1.- Composición de la bellota, la hierba y el pienso utilizados en la alimentación de los animales experimentales.

\begin{tabular}{|c|c|c|c|c|}
\hline & & Bellota & Hierba & $\begin{array}{r}\text { Pienso } \\
\text { experimental }\end{array}$ \\
\hline Materia Seca & $\mathrm{g} \mathrm{kg}^{-1}$ & 519,4 & 244 & 881,8 \\
\hline Proteína Bruta & $\mathrm{g} \mathrm{kg}^{-1} \mathrm{MS}$ & 80,1 & 158,2 & 140 \\
\hline Grasa Bruta & $\mathrm{g} \mathrm{kg}^{-1} \mathrm{MS}$ & 52,9 & 46,5 & 74,3 \\
\hline Fibra Bruta & $\mathrm{g} \mathrm{kg}^{-1} \mathrm{MS}$ & 37,4 & 210 & 42,7 \\
\hline Cenizas & $\mathrm{g} \mathrm{kg}^{-1} \mathrm{MS}$ & 33,6 & 73 & 62,7 \\
\hline $\mathrm{ELNs}^{1}$ & $\mathrm{~g} \mathrm{~kg}^{-1} \mathrm{MS}$ & 795,7 & 512,3 & 680,2 \\
\hline $\mathrm{C} 12: 0^{2}$ & $\%$ & 0,10 & 0,21 & 1,68 \\
\hline C14:0 ${ }^{2}$ & $\%$ & 0,24 & 0,54 & 0,67 \\
\hline$C-16: 0^{2}$ & $\%$ & 13,53 & 14,03 & 12,57 \\
\hline C16:1 (n-9) ${ }^{2}$ & $\%$ & 0,40 & 1,93 & 0,27 \\
\hline C17:0 & $\%$ & 0,21 & 0,20 & 0,07 \\
\hline C17:1 & $\%$ & 0,19 & 3,94 & 0,08 \\
\hline C18:0 ${ }^{2}$ & $\%$ & 1,54 & 3,18 & 5,07 \\
\hline C18:1(n-9) $)^{2}$ & $\%$ & 56,73 & 12,85 & 57,05 \\
\hline $\mathrm{C} 18: 2(\mathrm{n}-6)^{2}$ & $\%$ & 25,07 & 10,22 & 21,11 \\
\hline $\mathrm{C} 18: 3(n-3)^{2}$ & $\%$ & 1,71 & 52,20 & 1,13 \\
\hline C20:0 & $\%$ & 0,28 & 0,69 & 0,30 \\
\hline
\end{tabular}

${ }^{1}$ ELNs (Extractivos Libres de Nitrógeno).

${ }^{2}$ C12:0 (ácido laúrico) C14:0 (ácido mirístico) C16:0 (ácido palmítico),C16:1 (n-9)(ácido palmitoleico),C18:0(ácido esteárico),C18:1 (n-9)(ácido oleico),C18:2 (n-6)(ácido linoleico), C18:3 (n-3) (ácido linolénico). 
Para la determinación de la grasa intramuscular, se llevó a cabo un procedimiento basado en la extracción de la misma mediante hidrólisis (Folch et al., 1957). Posteriormente se determinó la composición de ácidos grasos mediante su extracción por el método de Bligh y Dyer (1959) y la posterior metilación para su inyección en un cromatógrafo de gases HP-6890 según la técnica descrita por López- Bote et al. (1997). Para ello se utilizó una columna capilar de polietilen glicol $30 \mathrm{~m}$ x 0,32 $\mathrm{mm}$ x 0,25 $\mu \mathrm{m}$, con un programa de temperatura desde $\operatorname{los} 170$ a $\operatorname{los} 245^{\circ} \mathrm{C}$. El inyector y el detector se mantuvieron a $250^{\circ} \mathrm{C}$ y el flujo del gas portador (helio) fue de $2 \mathrm{ml} / \mathrm{min}$.

\section{$\underline{\text { Análisis estadístico }}$}

Para estudiar el efecto de la alimentación sobre el contenido en grasa intramuscular y la composición en ácidos grasos de la paleta y el lomo se realizó un Análisis de Varianza utilizando el procedimiento GLM del paquete estadístico SAS versión 9.1 (2002).

\section{RESULTADOS}

Los diferentes métodos de alimentación (cebo $v s$ montanera) a los que fueron sometidos los cerdos ibéricos determinaron diferencias significativas tanto en el contenido en grasa intramuscular como en ácidos grasos de los lomos y las paletas curadas.

En relación a la grasa intramuscular, los datos que obtuvimos fueron muy diferentes para cada pieza, y dentro de cada pieza para cada tipo de alimentación. En los lomos, el contenido en grasa intramuscular fue significativamente mayor $(\mathrm{P}<0,05)$ para los lomos de montanera, con un $25,39 \%$, mientras que para los de cebo fue de un $16,65 \%$ (resultados expresados en materia seca). Las paletas también mostraron diferencias estadísticamente significativas respecto a su contenido en grasa intramuscular $(\mathrm{P}<0,05)$, los animales alimentados en montanera presentaron un $39,7 \%$ de grasa intramuscular mientras que para los alimentados con piensos fue significativamente menor $(29,1 \%)$. Al comparar los lomos con las paletas, el contenido en grasa intramuscular fue más elevado en paletas que en lomos, tanto en cebo como en montanera.

Para valorar los ácidos grasos presentes en cada pieza, nos centramos en los cuatro más representativos e importantes: ácido palmítico (16:0), ácido esteárico (18:0), ácido oleico (18:1 n-9) y ácido linoleico (18:2 n-6). En lomos, el contenido en ácido palmítico fue 
estadísticamente mayor en los animales alimentados con pienso durante su periodo de cebo que en aquellos alimentados en montanera. Mientras que el ácido oleico fue estadísticamente menor en los animales de cebo que en montanera, obteniéndose valores similares para el ácido linoleico para ambos tipos de alimentación (Tabla 2).

Tabla 2. Composición en ácidos grasos (g/100 g total de ácidos grasos) de la grasa intramuscular del lomo según la alimentación recibida por los animales experimentales.

\begin{tabular}{lcccc}
\hline & Montanera & Cebo & RMSE $^{1}$ & Valor P \\
\hline C12:0 & 0,08 & 0,08 & 0,007 & 0,029 \\
C14:0 & 1,32 & 1,42 & 0,105 & 0,046 \\
C16:0 & 21,99 & 23,66 & 0,954 & 0,003 \\
C16:1 n-9 & 3,57 & 4,20 & 0,341 & 0,000 \\
C17:0 & 0,17 & 0,17 & 0,027 & 0,941 \\
C17:1 & 0,19 & 0,20 & 0,032 & 0,264 \\
C18:0 & 8,76 & 9,41 & 0,957 & 0,107 \\
C18:1 n-9 & 53,57 & 50,86 & 1,520 & 0,000 \\
C18:2 n-6 & 8,56 & 8,53 & 1,308 & 0,958 \\
C18:3 n-3 & 0,59 & 0,39 & 0,124 & 0,009 \\
C20:0 & 0,17 & 0,13 & 0,025 & 0,005 \\
C21 :0 & 1,04 & 0,93 & 0,129 & 0,056 \\
AGS & 33,52 & 35,81 & 1,673 & 0,002 \\
AGM $^{3}$ & 57,33 & 55,27 & 1,609 & 0,004 \\
AGP $^{4}$ & 9,15 & 8,93 & 1,351 & 0,690 \\
\hline
\end{tabular}

${ }^{1}$ RMSE: Raíz Cuadrada del Cuadrado medio del Error.

${ }^{2}$ AGS: Sumatorio de ácidos grasos saturados.

${ }^{3}$ AGM: Sumatorio de ácidos grasos monoinsaturados.

${ }^{4}$ AGP: Sumatorio de ácidos grasos poliinsaturados.

En las paletas encontramos diferencias estadísticamente significativas en el ácido palmítico, con valores de $21,81 \%$ y $20,75 \%$ en cebo y montanera respectivamente; y en el ácido linoleico, con valores de $9,02 \%$ y $10,57 \%$ en cebo y montanera respectivamente (Tabla 3 ). 
Tabla 3. Composición en ácidos grasos (g/100 g total de ácidos grasos) de la grasa intramuscular de la paleta según la alimentación recibida por los animales experimentales.

\begin{tabular}{|c|c|c|c|c|}
\hline & Montanera & Cebo & $\mathrm{RMSE}^{1}$ & Valor $\mathbf{P}$ \\
\hline C12:0 & 0,08 & 0,08 & 0,011 & 0,971 \\
\hline C14:0 & 1,35 & 1,37 & 0,134 & 0,742 \\
\hline C16:0 & 20,75 & 21,81 & 0,625 & 0,001 \\
\hline C16:1 n-9 & 2,89 & 3,41 & 0,214 & 0,000 \\
\hline C17:0 & 0,20 & 0,19 & 0,028 & 0,398 \\
\hline C17:1 & 0,21 & 0,23 & 0,019 & 0,033 \\
\hline C18:0 & 7,44 & 7,75 & 0,439 & 0,119 \\
\hline C18:1 n-9 & 54,37 & 54,51 & 0,835 & 0,695 \\
\hline C18:2 n-6 & 10,57 & 9,02 & 0,850 & 0,000 \\
\hline C18:3 n-3 & 0,79 & 0,38 & 0,066 & 0,001 \\
\hline C20:0 & 0,12 & 0,11 & 0,014 & 0,020 \\
\hline C21:0 & 1,23 & 1,15 & 0,070 & 0,017 \\
\hline $\mathbf{A G S}^{2}$ & 31,17 & 32,45 & 0,772 & 0,001 \\
\hline $\mathbf{A G M}^{3}$ & 57,47 & 58,15 & 0,846 & 0,077 \\
\hline $\mathbf{A} \mathbf{G} \mathbf{P}^{4}$ & 11,36 & 9,40 & 0,878 & 0,000 \\
\hline
\end{tabular}

${ }^{1}$ RMSE: Raíz Cuadrada del Cuadrado medio del Error.

${ }^{2}$ AGS: Sumatorio de ácidos grasos saturados.

${ }^{3}$ AGM: Sumatorio de ácidos grasos monoinsaturados.

${ }^{4}$ AGP: Sumatorio de ácidos grasos poliinsaturados.

Tanto en lomos, como en paletas apreciamos un mayor contenido de ácido linolénico (C18:3 n-3) en aquellos animales alimentados con bellotas y pasto.

Al comparar lomos y paletas, los ácidos grasos saturados (ácido palmítico y ácido esteárico principalmente), fueron más abundantes en lomo que en paleta, presentando diferencias estadísticamente significativas el ácido palmítico en ambos productos al comparar cebo con montanera. El ácido oleico mostró diferencias estadísticamente significativas en lomo, pero no en paleta, al contrario que lo que sucedió con el ácido linoleico, que presentó diferencias estadísticamente significativas en paleta. 


\section{DISCUSIÓN}

La alimentación influye de forma directa sobre la cantidad y calidad de la grasa en el cerdo, y por ello, los resultados entre los distintos trabajos pueden variar. Si comparamos la composición de la bellota de nuestro estudio con el realizado por Soto et al. (2008), el ácido linoleico en bellotas es mayor en nuestro trabajo (25,07\% frente a $18,01 \%)$, esto es debido a que alimentamos nuestros animales con bellotas de alcornoques mayoritariamente, mientras que en el estudio mencionado las bellotas procedían de encinas. Este hecho es también novedoso en nuestro experimento, ya que hay zonas de dehesa diferentes a otras y por ello existe tanta heterogeneidad en el producto final, aunque todos los animales estén alimentados en montanera, la composición de los alimentos varía de forma ostensible dependiendo de la diferencia de arbolado y pasto en la dehesa. Existen otros estudios, como el realizado por Isabel y González (2008) que corroboran la diferente composición en ácidos grasos de las bellotas de encina respecto a las de alcornoque, en el trabajo mencionado podemos observar como los ácidos esteárico y oleico fueron mayores en bellotas de encina $(3,61 \pm 0,79 \%$ y $59,53 \pm 4,95 \%$ respectivamente) (media \pm sd) y menores en bellotas de alcornoque $(1,8 \pm 0,34 \%$ y $52,28 \pm 9,08 \%$ respectivamente). Al contrario ocurrió con el ácido linoleico, siendo menor en bellotas de encina $(19,9 \pm 3,9 \%)$ y mayor en bellotas de alcornoque $(26,7 \pm 6,73 \%)$.

El contenido en grasa intramuscular fue más elevado en paletas que en lomos, esto es debido a que la paleta en sí, es más grasa que el lomo, tanto en fresco como tras ser sometida a un proceso de curación (Ramírez y Cava, 2008).

Los animales con alimentación en cebo consumieron un pienso con un porcentaje de grasa superior a los alimentados en régimen de montanera, pero el consumo de los animales en uno y en otro sistema varía. Mientras los animales en régimen de cebo recibieron un total de 400 $\mathrm{kg}$ de pienso por animal (3,3 kg pienso/día) lo que supone una ingesta aproximada de $29,3 \mathrm{~kg}$ de grasa $(0,074 \mathrm{~kg}$ grasa $/ \mathrm{kg}$ pienso), los alimentados en régimen extensivo en base a pasto y bellotas consumieron una media de $8 \mathrm{~kg}$ de bellota diaria lo que supone un total de $50,8 \mathrm{~kg}$ de grasa $(0,053 \mathrm{~kg}$ grasa/kg bellota). Isabel y González (2008) y Petrón et al. (2004) observaron que existe una relación entre la composición de la bellota y el pasto y la calidad de los productos cárnicos, su composición en ácidos grasos y grasa intramuscular, apuntando los mismos resultados que describimos en nuestro estudio. 
Al final del periodo de cebo, los animales alimentados con pienso consumieron menos grasa por lo que aumentó la síntesis endógena aumentando así los ácidos grasos saturados (ácido palmítico y ácido esteárico).

Dentro de los ácidos grasos, el mayoritario fue el ácido oleico, debido a la gran cantidad de este ácido oleico que poseen las bellotas, si es que hablamos de animales alimentados en montanera; y si nos referimos a animales de cebo, se debe a que el pienso era rico en ácido oleico. Estos resultados son similares a los descritos por Cava et al. (1997), donde, al igual que en nuestro estudio, el ácido palmítico es mayor en cerdos alimentados en cebo que en montanera tanto en paletas como en lomos; y los ácidos oleico y linoleico son mayoritarios en montanera tanto para lomos como para paletas.

\section{CONCLUSIONES}

Los resultados de nuestro estudio demuestran que la bellota proporciona una cantidad abundante de grasa al cerdo ibérico, lo cual aumenta el contenido en grasa intramuscular de los lomos y paletas curados. Además la bellota de alcornoque es una fuente de ácido oleico y linoleico, por lo que durante su estancia en montanera se produce un aumento de la concentración de estos ácidos grasos en sus tejidos y en los productos cárnicos derivados a partir de ellos.

\section{AGRADECIMIENTOS}

El presente trabajo de investigación se ha realizado gracias al proyecto de investigación (artículo 83 LOU) 35/2012 Universidad Complutense de Madrid.

\section{BIBLIOGRAFÍA}

Aparicio, J.B. (1987). El cerdo ibérico. Sánchez Romero Carvajal, Jabugo S. A. Huelva. I. Gráficas Gaditanas.

Bligh, E.G. y Dyer,W.J. (1959). A rapid method for total lipid extraction and purification. Canadian Journal of Biochemistry and Physiology 37 (8): 911-7. 
Cava, R., Ruiz, J., López-Bote, C., Martín, L., García, C., Ventanas, J., Antequera, T. (1997). Influence of finishing diet on fatty acid profiles of intramuscular lipids, triglycerides and phospholipids in muscles of the Iberian pig. Meat Science 45 (2): 263-70.

Folch, J., Lees, M., Sloane-Stanley, G.H. (1957). A simple method for isolation and purification of total lipids from animal tissues. Journal of Biological Chemistry 226: 497509.

Isabel, B. (2000). Incorporación tisular de ácidos grasos, modificación en la consistencia de la grasa y susceptibilidad a la oxidación por la utilización de distintos tipos de grasas y antioxidantes naturales en la alimentación del cerdo. Tesis Doctoral, U.C.M.

Isabel, B y González, J.L. (2008). Valoración nutritiva de la bellota en la montanera 20072008. Repercusiones en la gestión de la dehesa. Sólo Cerdo Ibérico 19: 65-90.

Lopez-Bote, C., Rey, A., Isabel, B., Sanz Arias, R. (1997). Effect of feeding diets high in monounsaturated fatty acids and alpha-tocopheryl acetate to rabbits on resulting carcass fatty acid profile and lipid oxidation. Animal Science 64: 177-186.

Lopez-Bote, C. (1998). Sustained utilization of the Iberian pig breed. Meat Science 49: S17S27.

Lopez-Bote, C.J. (2000). Dietary treatment and quality characteristics in Mediterranean meat products. In Antioxidants in muscle food. Massachusetts: Ed. E. Decker, C. Faustman, C.J. Lopez-Bote, 2000, 345-366.

Petrón, M.J., Muriel, E., Timón, ML., ， L., Antequera, T. (2004). Fatty acids and triacylglycerols profiles from different types of Iberian dry-cured hams. Meat Science 68 (1): 71-77.

Ramírez, R y Cava, R. (2008). Changes in fatty acid composition of 2 muscles from 3 Iberian x Duroc genotypes after refrigerated storage. Food Science and Technology International 14: $127-138$.

Rey, A. I., Lopez-Bote, C. J, Sanz Arias, R. (1997). Effect of extensive feeding on tocopherol concentration and oxidative stability of muscle microsomes from Iberian Pigs. Animal Science 65 (5): 15-520. 
Soto, E., Hoz, L., Ordóñez, J.A., Hierro, E., Herranz, B., López-Bote, C., Cambero, M.I. (2008). Impact of feeding and rearing systems of Iberian pigs on volatile profile and sensory characteristics of dry-cured loin. Meat Science 79: 666-676.

Vázquez, F., Doncel, E., Ramos, S. (2000). Valoración de montaneras: Producción frutal y evolución de la calidad. II Jornadas del cerdo Ibérico y sus productos, 15-29. 